\title{
Systemic Delivery of NAC-1 siRNA by Neuropilin-Targeted Polymersomes Sensitizes Antiangiogenic Therapy of Metastatic Triple Negative Breast Cancer
}

Hongyu Wang ${ }^{1}$, Xiaohui Wang ${ }^{2}$, Yi Zhang ${ }^{2, *}$, Ru Cheng, ${ }^{1, *}$, Jiandong Yuan ${ }^{3}$, and Zhiyuan Zhong ${ }^{1, *}$

${ }^{1}$ Biomedical Polymers Laboratory, College of Chemistry, Chemical Engineering and Materials Science, and State Key Laboratory of Radiation Medicine and Protection, Soochow University, Suzhou, 215123, P. R. China.

${ }^{2}$ Department of Pharmacology, College of Pharmaceutical Sciences, Soochow University, Suzhou, 215123, P. R. China.

${ }^{3}$ BrightGene Bio-Medical Technology Co., Ltd., Suzhou 215123, PR China.

* Corresponding authors. Tel/Fax: +86-512-65880098, Email: zhangyi@ suda.edu.cn (Y. Zhang); rcheng@suda.edu.cn (R. Cheng); zyzhong@suda.edu.cn (Z. Zhong). 


\section{Materials}

Trimethylene carbonate (TMC, Jinan Daigang Biomaterial, China) was recrystallized twice in anhydrous toluene. $\mathrm{N}, \mathrm{N}$-dimethylformamide (DMF) was refluxed over $\mathrm{CaH}_{2}$ and distilled prior to use. tLyP-1 peptide (CGNKRTR, 99.4\%, Chinapeptide), maleimide-poly(ethylene glycol) $\left(\right.$ Mal-PEG-OH, $M_{\mathrm{n}}=7.5 \mathrm{~kg} / \mathrm{mol}$, Jenkem Technology), glutathione (GSH, 98\%, Amresco, USA), HEPES (Amresco, USA), TAE (Tiandz, China), bevacizumab (Roche, USA), BCA protein assay kit (Pierce, USA), spermine (Fluka, USA), ethidium bromide (Abcam, Shanghai, China), DNA loading buffer (Biosharp, Hefei, China), 4',6-diamidino-2-phenylindole (DAPI, Invitrogen, USA), trypsin (Jinuo Biomedical Technology, China), fetal bovine serum (FBS, Gibco, USA), Dulbecco's modified Eagle's medium (DMEM, Gibco, USA), penicillin-streptomycin (Jinuo Biomedical Technology, China), Trizol reagent (Tiangen, Beijing, China), MicroRNA Reverse Transcription Kit (TaKaRa, Japan), TB Green (TaKaRa, Japan), RIPA lysis buffer (Beyotime Biotechnology, Shanghai, China), bromophenol blue (Beyotime Biotechnology, Shanghai, China), polyvinylidene fluoride membrane (Millipore, USA), the anti-NAC-1 antibody (CST, USA), antiGAPDH antibody (Servicebio, Wuhan, China), the secondary goat anti-rabbit antibody (Servicebio, Wuhan, China), matrigel coated trans-well insert chambers ( $8 \mu \mathrm{m}$, Corning, USA) and dichloromethane (DCM) were all used as received. Dithiolane trimethylene carbonate (DTC), PEG-P(TMC-co-DTC)-spermine and Mal-PEG-P(TMC-co-DTC) were prepared according to our earlier woks. ${ }^{40,} 41$ siNAC-1 (Sense: 5'UGAUGUACACGUUGGUGCCUGUCACCA-3', Antisense: 5'UGGUGACAGGCACCAACGUGUACAUCA-3'), siScramble (siSCR, Sense: 5'UUGGUCUUGCCAUAUAGCACCGCAGGU-3', Antisense: 5'AACCAGAACGGUAUAUCGUGGCGUCCA-3'), and Cy5-labeled siSCR (Cy5siSCR) were bought from Sangon Biotech., China.

\section{Characterization}

${ }^{1} \mathrm{H}$ NMR spectra were recorded on a Unity Inova 400 spectrometer (Agilent, USA) operating at $600 \mathrm{MHz}$ using chloroform-d ( $\mathrm{CDCl}_{3}$, Andover, MA, USA) as a solvent. The tLyP-1 functionality was determined by BCA protein assay kit (Pierce, USA) as described by the manufacturer. The PB solutions of free tLyP-1 peptide with varying concentrations were used for setting up the standard curve. The PB solution of tLyP-1- 
Ps self assembled from tLyP-1-PEG-P(TMC-co-DTC) copolymer with unknown tLyP1 amount were prepared and after mixing and incubating with BCA working reagents in microplates, the samples were measured at $562 \mathrm{~nm}$ using a plate reader. The PB solution was measured at $562 \mathrm{~nm}$ as a blank control. The polymersomes dispersion was incubated in a shaking bath (THZ-C, Taicang Instrument, China). The size of the polymersomes was determined using dynamic light scattering (DLS). Measurements were carried out at $25{ }^{\circ} \mathrm{C}$ using a Zetasizer Nano-ZS from Malvern Instruments equipped with a $633 \mathrm{~nm}$ He-Ne laser using back-scattering detection. Transmission electron microscopy (TEM) was performed using a Tecnai G220 TEM operated at an accelerating voltage of $200 \mathrm{kV}$. The amount of siRNA loaded into the polymersomes was determined by Nanodrop 2000 UV-Vis spectrophotometer (Thermo Fisher Scientific, USA) at the absorbance ratio of 260/280. Gel images were recorded by Chem Studio imaging system (Analytik Jena AG). The confocal laser scanning microscopy (CLSM) images of cells were taken on a Leica TCS SP5. Flow cytometry was conducted on a flow cytometer (FACS Calibur, BD Biosciences, USA). qRT-PCR was carried out with an ABI 7500 Fast Real-Time PCR System (Life technologies, USA). Images of transwell and wound healing assays were taken with Nikon Eclipse Ti microscope adjusted with a Nikon digital sight camera. Fluorescence spectra were recorded using a FLS920 fluorescence spectrometer. In vivo and ex vivo fluorescence images were acquired using near-infrared fluorescence imaging system (IVIS, Lumina II; Caliper, MA). H\&E images were acquired using a digital microscope (Olympus BX41).

\section{Extraction of RNA and mRNA expression analysis}

The cells were washed with PBS and the total RNA was extracted using Trizol reagent following the protocols of the manufacturer. For mRNA expression analysis, $0.5 \mathrm{mg}$ of total RNA was applied for reverse transcription to synthesize cDNA of mRNA using MicroRNA Reverse Transcription Kit. qRT-PCR analysis was performed using TB Green and special RT-primer designed for NAC-1 according to the manufacturer's instructions. $20 \mu \mathrm{L}$ of the reverse transcription mix was amplified by PCR with the following program: $95{ }^{\circ} \mathrm{C}$ for $0.5 \mathrm{~min}, 40$ cycles at $95{ }^{\circ} \mathrm{C}$ for $5 \mathrm{~s}$ and 60 ${ }^{\circ} \mathrm{C}$ for $0.5 \mathrm{~min}$. GAPDH was used as an endogenous control for normalization. The mRNA expression level was calculated based on comparative $2^{-\Delta \Delta \mathrm{Ct}}$ method and 
normalized to that obtained from non-treated cells. Results are expressed as mean \pm standard deviation $(\mathrm{n}=4)$. NAC-1 primer sequences were as follows: forward: 5'AAGCTGAGGATCTGCTGGAA-3' and 5'CCAGACACTGCAGATGGAGA-3'.

\section{Protein collection and expression analysis}

Cultured cells were harvested and lysized with RIPA lysis buffer by incubation on ice for $15 \mathrm{~min}$. After centrifugation at 12,000 rpm for $15 \mathrm{~min}$, the supernatants were collected and the concentrations of proteins were measured using BCA protein assay kit. The protein samples were denatured by boiling for $5 \mathrm{~min}$ with bromophenol blue and loaded onto SDS-PAGE gel for electrophoresis. After transferring to a polyvinylidene fluoride membrane, the membranes were then incubated in the blocking solution (5\% non-fat dried milk) at room temperature for $1 \mathrm{~h}$. The anti-NAC-1 antibody and anti-GAPDH antibody were respectively added into blocking solution and incubated at $4{ }^{\circ} \mathrm{C}$ overnight. The membranes were subsequently incubated with the secondary goat anti-rabbit antibody for $90 \mathrm{~min}$. The membrane was washed with Trisbuffered saline/0.1\% Tween-20 followed by incubation with secondary antibody. Protein expression was normalized against GAPDH expression. Blotting images were acquired with the Odyssey infrared imaging system and analyzed by Image $\mathbf{J}$ software.

\section{Invasion assay}

For invasion assay, cells were seeded over the Matrigel coated trans-well insert chambers and $750 \mu \mathrm{L}$ chemo-attractant (20\% FBS in DMEM) was added to the basal chamber. The cells were incubated for $24 \mathrm{~h}$ at $37{ }^{\circ} \mathrm{C}$ and $5 \% \mathrm{CO}_{2}$. After $24 \mathrm{~h}$, media were removed carefully and the cells were washed with PBS twice. Cells in the upper chambers were removed by wiping with a cotton swab and cells invaded to the lower surface of filter were fixed in $4 \%$ paraformaldehyde for $15 \mathrm{~min}$, stained with $0.2 \%$ crystal violet for 15 min and imaged using a Nikon Eclipse Ti microscope adjusted with a Nikon digital sight camera. Images were processed using Image $\mathbf{J}$ software.

\section{Cell culture}


Triple-negative breast cancer cell line, MDA-MB-231, was maintained in Dulbecco's Modified Eagle's Medium (DMEM) supplemented with 10\% FBS and 1\% penicillin-streptomycin at $37{ }^{\circ} \mathrm{C}$ in a humidified atmosphere with $5 \% \mathrm{CO}_{2}$.

\section{Pharmacokinetics}

Mice were handled according to the protocols approved by Laboratory Animal Center and Animal Care and Use Committee of Soochow University. For the pharmacokinetics study, tLyP-1-Ps-Cy5-siSCR or Ps-Cy5-siSCR (Cy5-siSCR dosage: $2 \mathrm{mg} / \mathrm{kg}$ ) was intravenously injected to female BALB/c mice weighed around $20 \mathrm{~g}$ (n $=3$ ). Blood samples were collected into heparinized tubes from the eye socket of mice at different time points $(0.08,0.17,0.25,0.5,1,2,4,8,12$ and $24 \mathrm{~h})$ and immediately centrifuged at $3000 \mathrm{rpm}$ for $15 \mathrm{~min}$ to take out $15 \mu \mathrm{L}$ of plasma. Cy5-siSCR in the plasma was extracted using $700 \mu \mathrm{L}$ DMSO for 1 day in the dark followed by centrifugation and fluorescence measurement of the supernatant. The blood circulation half-life was calculated by PKSolver software with a 2-compartment model.

\section{Statistical analysis}

All data were presented as average \pm SD. They were compared using Student's ttest for two sample comparison or one-way analysis of variance (ANOVA) for multiple comparisons. ${ }^{*} \mathrm{p}<0.05, * * \mathrm{p}<0.01$, and $* * * \mathrm{p}<0.001$.

Table S1. Characterization of tLyP-1-Ps-Cy5-siSCR in PB (10 mM, pH 7.4, 2 $\mathrm{mg} / \mathrm{mL}$, theoretical DLC $=9.1 \mathrm{wt} . \%)$.

\begin{tabular}{ccccccc}
\hline Entry & $\begin{array}{c}\text { tLyP-1 density } \\
(\%)\end{array}$ & $\begin{array}{c}\text { Size }^{\mathrm{a}} \\
(\mathrm{nm})\end{array}$ & $\mathrm{PDI}^{\mathrm{a}}{ }^{\mathrm{DLE}}$ & $\begin{array}{c}\mathrm{DLC}^{\mathrm{b}} \\
(\%)\end{array}$ & $\begin{array}{c}\text { Zeta }^{\mathrm{c}} \\
(w t . \%)\end{array}$ & $(\mathrm{mV})$ \\
\hline 1 & 0 & 58.9 & 0.10 & 82 & 7.5 & -1.7 \\
2 & 10 & 47.1 & 0.12 & 86 & 7.8 & -3.4 \\
3 & 20 & 47.5 & 0.14 & 87 & 7.9 & -5.7 \\
4 & 30 & 71.3 & 0.11 & 69 & 6.3 & -8.1 \\
\hline
\end{tabular}

${ }^{\mathrm{a}}$ Determined by DLS in $5 \mathrm{mM}$ PB $(\mathrm{pH}=7.4) .{ }^{\mathrm{b}}$ Determined by Nanodrop 2000c UV-Vis spectrophotometer in $5 \mathrm{mM} \mathrm{PB}(\mathrm{pH}=7.4) .{ }^{\mathrm{c}}$ Determined by electrophoresis $\left(25^{\circ} \mathrm{C}\right)$ 
A
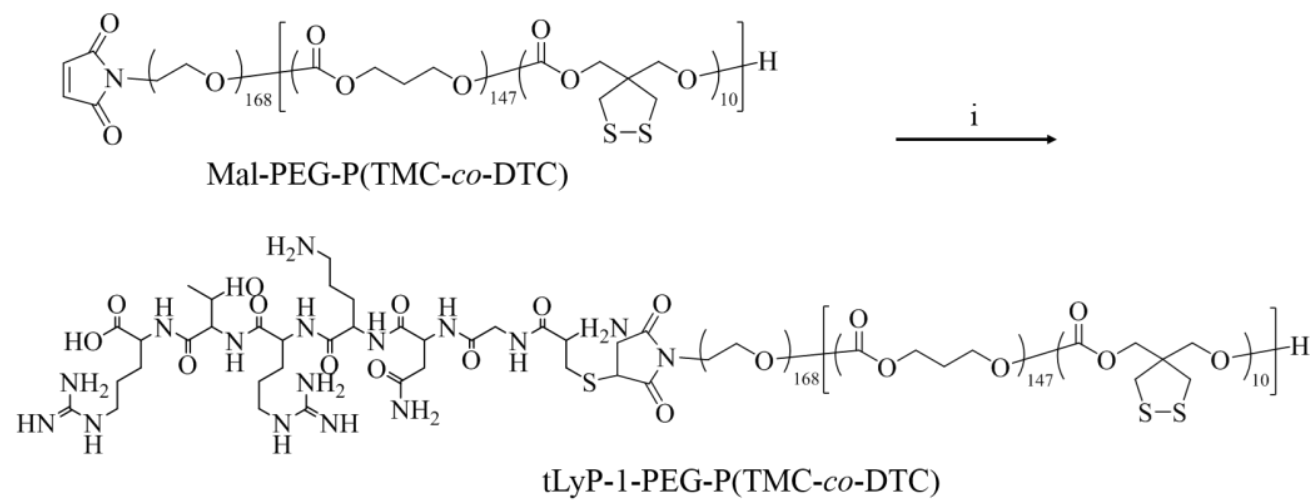

B

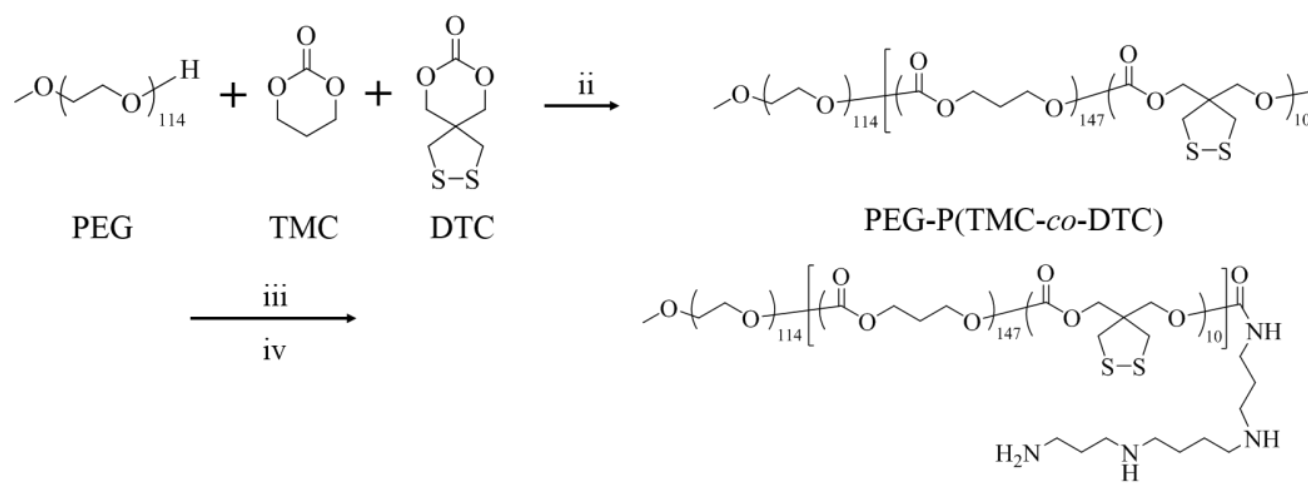

PEG-P(TMC-co-DTC)-spermine

Scheme S1. Synthesis of tLyP-1-PEG-P(TMC-co-DTC) (A) and PEG-P(TMC-coDTC)-spermine (B). Conditions: i) tLyP-1-SH, DMF, $30{ }^{\circ} \mathrm{C}, 12 \mathrm{~h}$; ii) DPP, $\mathrm{CH}_{2} \mathrm{Cl}_{2}, 30^{\circ} \mathrm{C}$, $96 \mathrm{~h}$; iii) CDI, $\mathrm{CH}_{2} \mathrm{Cl}_{2}, 30^{\circ} \mathrm{C}, 4 \mathrm{~h}$; iv) spermine, DMF, $0{ }^{\circ} \mathrm{C}, 24 \mathrm{~h}$. 
A

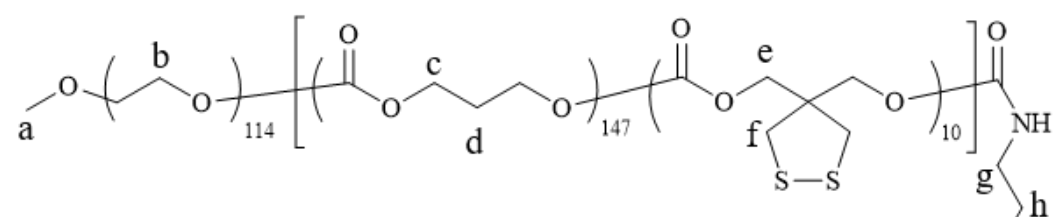
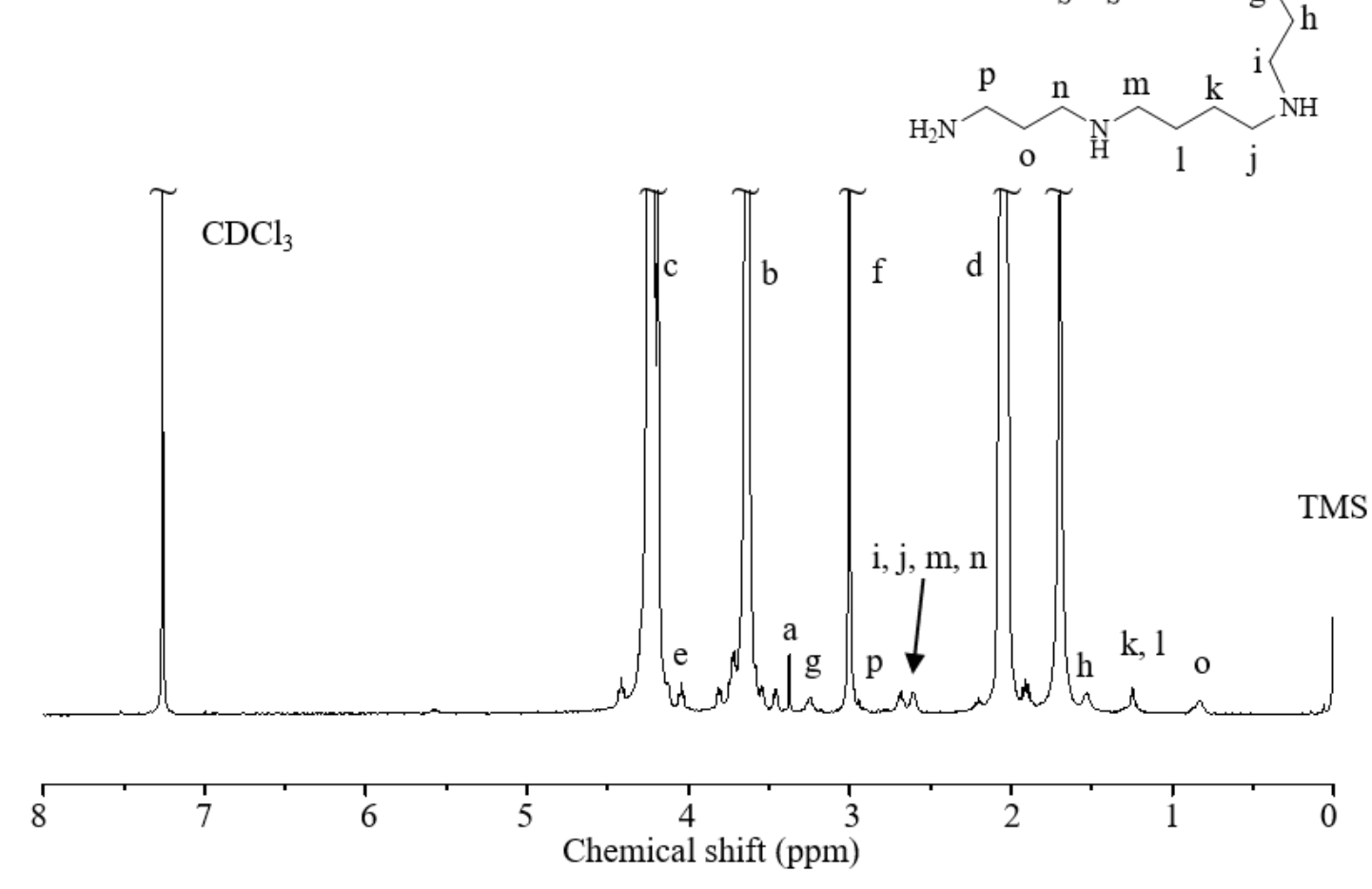

B
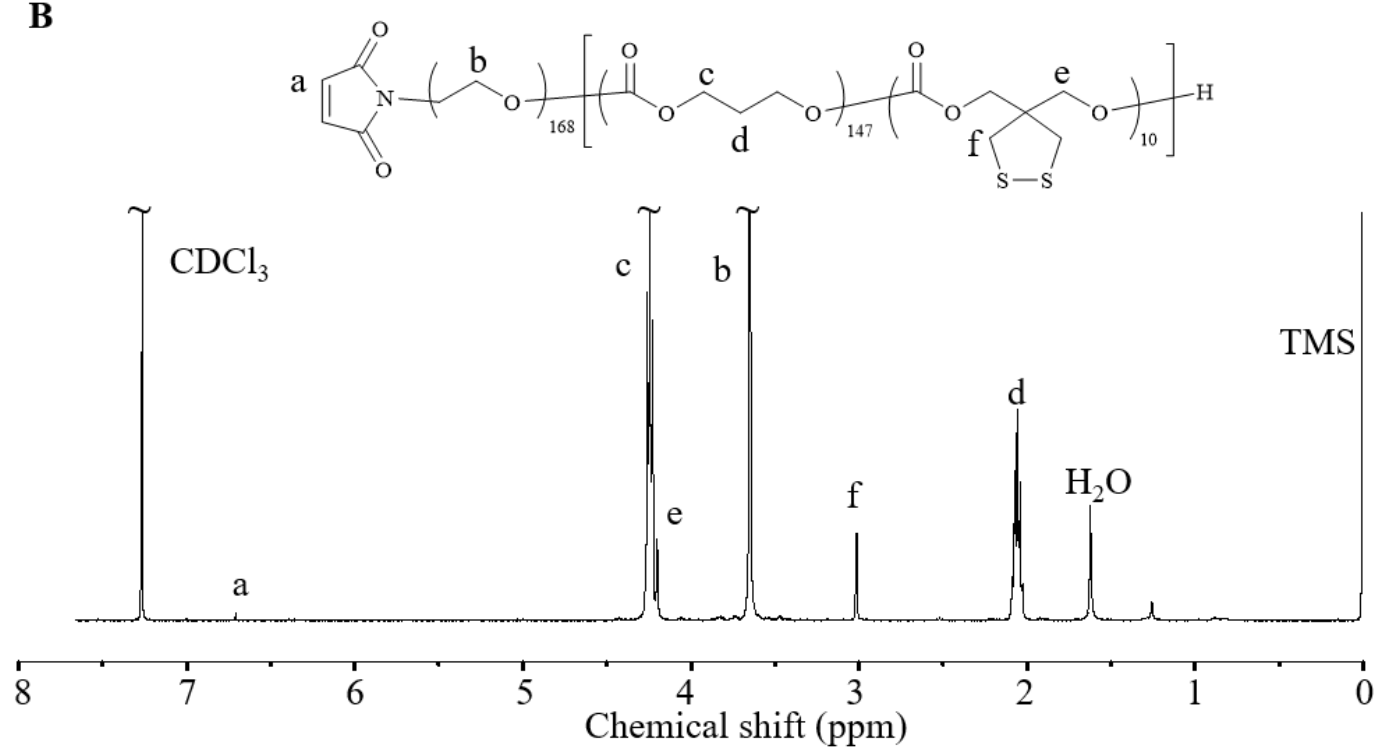


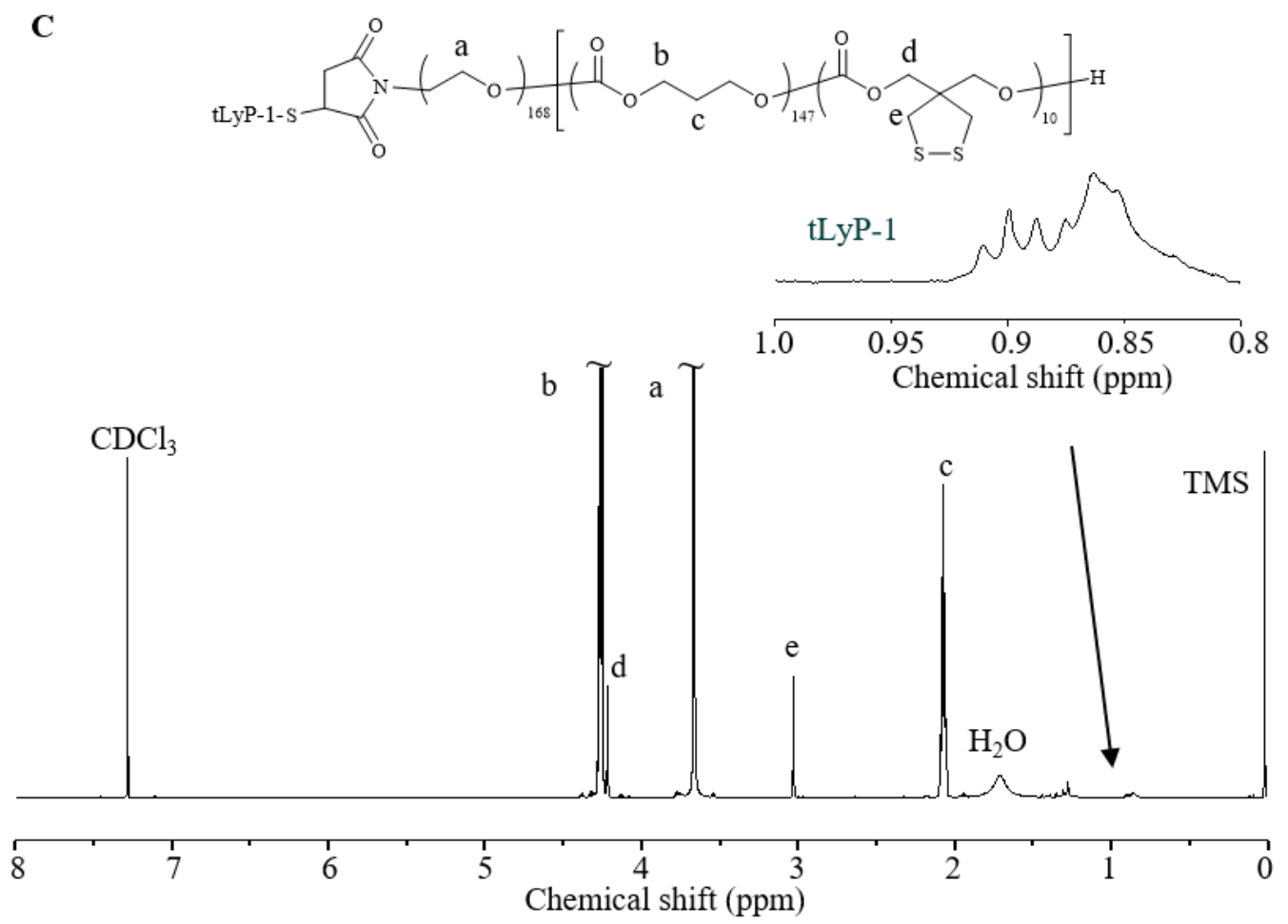

Figure S1. ${ }^{1} \mathrm{H}$ NMR spectra $\left(600 \mathrm{MHz}, \mathrm{CDCl}_{3}\right)$ of PEG-P(TMC-co-DTC)-spermine (A), Mal-PEG-P(TMC-co-DTC) (B) and tLyP-1-PEG-P(TMC-co-DTC) (C).
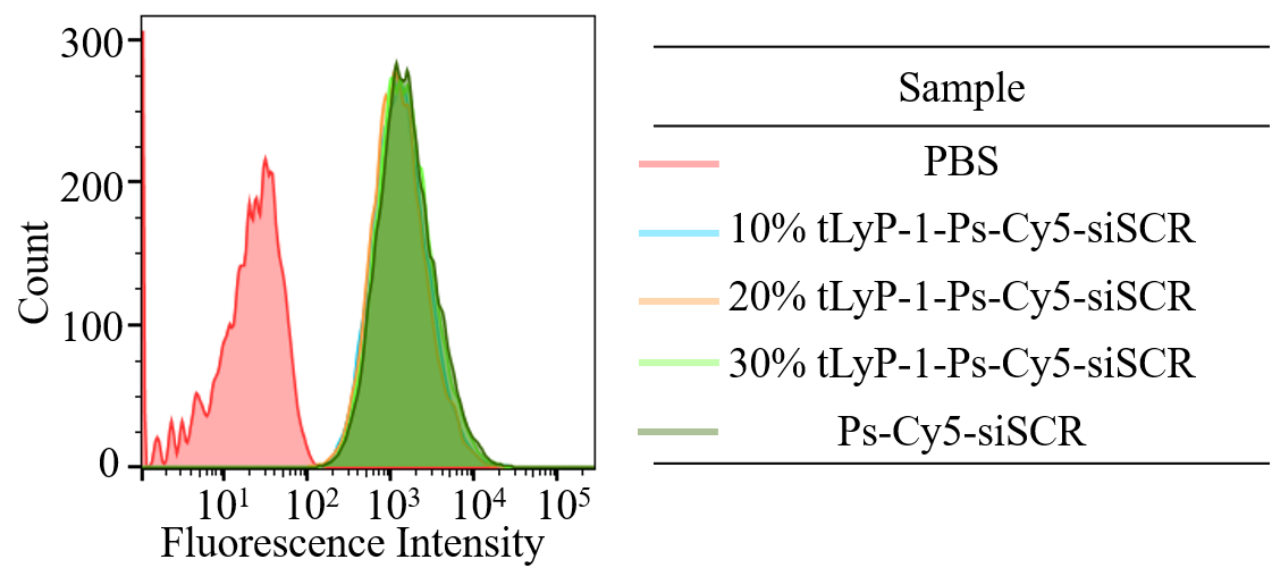

Figure S2. Flow cytometry of tLyP-1-Ps-Cy5-siSCR with varying tLyP-1 densities of $10 \mathrm{~mol} . \%, 20 \mathrm{~mol} \%$ and $30 \mathrm{~mol} . \%$ or Ps-Cy5-siSCR for $4 \mathrm{~h}$ in bEnd.3 cells (Cy5siSCR dosage: $100 \mathrm{nM})$. 


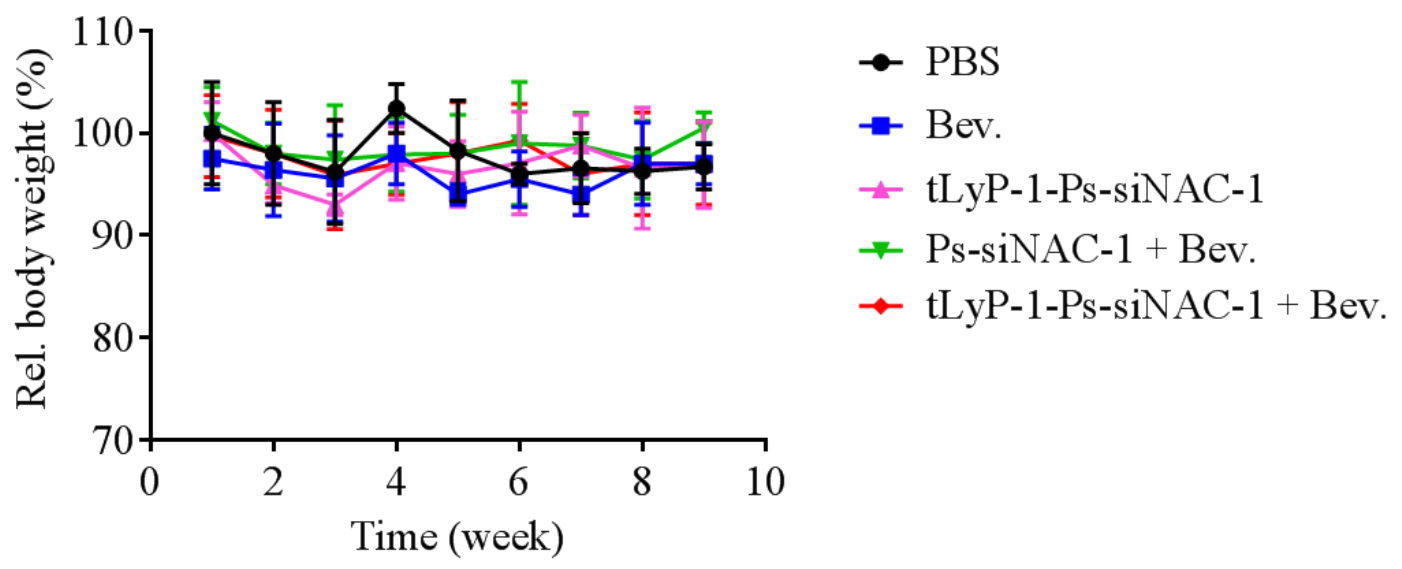

Figure S3. Body weight changes of mice within 9 weeks $(n=5)$. 